\title{
Statistical properties of radiation power levels from a high-gain free-electron laser at and beyond saturation
}

\author{
C.B. Schroeder, W.M. Fawley, and E. Esarey \\ Center for Beam Physics, Lawrence Berkeley National Laboratory, Berkeley, CA 94720, USA
}

\begin{abstract}
We investigate the statistical properties (e.g., shot-to-shot power fluctuations) of the radiation from a high-gain free-electron laser (FEL) operating in the nonlinear regime. We consider the case of an FEL amplifier reaching saturation whose shot-to-shot fluctuations in input radiation power follow a gamma distribution. We analyze the corresponding output power fluctuations at and beyond first saturation, including beam energy spread effects, and find that there are well-characterized values of undulator length for which the fluctuation level reaches a minimum.
\end{abstract}

\section{Introduction}

A detailed understanding of the statistical properties of the radiation produced at or near saturation by a high gain FEL based upon selfamplified spontaneous emission (SASE) is critical for generation of FEL radiation in both current experiments [1-3] and future proposed x-ray devices $[4,5]$. For example, the ability to describe, predict, and control shot-to-shot power fluctuations in the output radiation will be important for most user applications.

In this paper, we present numerical results concerning certain statistical properties of the radiation emitted by high-gain FEL's operating in the nonlinear regime at and beyond saturation. Our study is motivated by recent proposals $[6,7]$ to improve the output properties (e.g., spectral bandwidth or pulse duration) of high gain SASE FEL's. The basic scheme is to first use a SASE FEL to generate radiation, which would then be manipulated (by a monochromator or other optics) to serve as the input seed to a second undulator acting as a (nearly monochromatic) FEL amplifier. This method can be used both for monochromatization [6] and, when combined with the use of a chirped electron beam, for femtosecond pulse generation [7]. Our particular interest is to determine the optimal length for the second undulator to minimize pulse-topulse output power fluctuations (which arise naturally from the fluctuations associated with the monochromatized SASE radiation of the first undulator).

\section{FEL amplifier in the nonlinear regime}

In this section we examine the characteristics of the output radiation of an monochromatic FEL amplifier operating at and beyond saturation. Consider the dependence of the output radiation power versus undulator length for various input powers. Figure 1 shows the power $P$ versus location along the undulator, as obtained from onedimensional (1D) runs (i.e., no diffraction) using the GINGER simulation code [8] to model a monochromatic FEL amplifier with the following physical parameters: radiation wavelength $\lambda_{s}=$ $1 \mu \mathrm{m}$, beam current $I_{B}=1.0 \mathrm{kA}$, beam energy $E_{B}=204 \mathrm{MeV}(\gamma=400)$, no instantaneous energy spread, normalized emittance $\varepsilon_{N}=20 \pi$ mm-mrad, FEL parameter $\rho=6.4 \times 10^{-3}$, undulator period $\lambda_{u}=4.0 \mathrm{~cm}$, and normalized $\mathrm{rms}$ undulator strength $a_{u}=2.65$. The curves in Fig. 1 show the exponential power gain and saturation for three initial input radiation powers, $2.5,25$, and $250 \mathrm{~kW}$, and are nearly self-similar in shape and saturation power (a result that has been long known for $\left.P_{\text {in }} \ll P_{\text {sat }}\right)$. Once saturation is reached, the output radiation power then oscillates with $z$ at the synchrotron bounce period

$$
\tau_{b} \simeq \frac{\lambda_{u}}{2 c}\left(\frac{1+a_{u}^{2}}{a_{u} a_{s}}\right)^{1 / 2},
$$




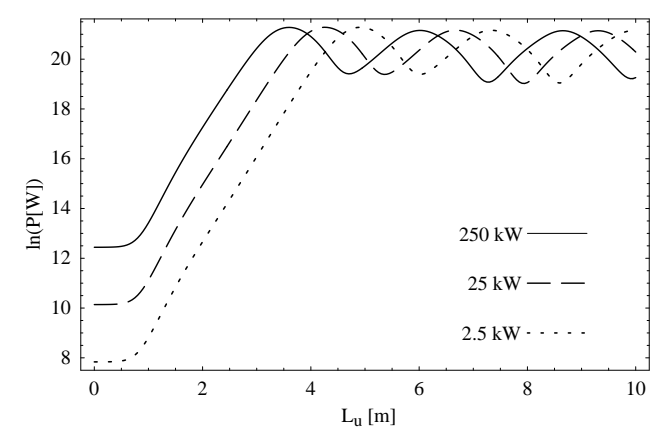

Figure 1. Radiation power $\ln (P[\mathrm{~W}])$ versus undulator length $L_{u}$ for several input powers.

where $a_{s}$ is the normalized radiation vector potential. The oscillations have near-perfect periodicity, indicating that most particles are deeplytrapped in the ponderomotive well where the bounce period is nearly constant.

Once the undulator length $L_{u}$ exceeds the length necessary to enter the saturated gain regime, we expect that the shot-to-shot power fluctuations will decrease in a relative sense. This behavior is confirmed by Fig. 2 where we plot the output radiation power as a function of the input radiation power (normalized to a nominal input power $P_{\text {in }}=25 \mathrm{~kW}$ ) for several different undulator lengths. If $L_{u}$ is much shorter (e.g., $L_{u}=3 \mathrm{~m}$ with $P_{\text {in }}=25 \mathrm{~kW}$ ) than the saturation length corresponding to the first maximum of output radiation power (e.g., $L_{\mathrm{sat}}=4.25 \mathrm{~m}$; see the dashed curve of Fig. 1), then there is a large variation of $P_{\text {out }}$ with $P_{\text {in }}$. As $L_{u}$ approaches the length necessary for saturation, the sensitivity to $P_{\text {in }}$ becomes much less, as shown by the solid curve of Fig. 2, $z=4.5 \mathrm{~m}$. Consequently, we conclude that in the presence of shot-to-shot input power fluctuations, higher stability of the output radiation power can be achieved by making the undulator length of a monochromatic FEL amplifier sufficiently long to operate in the saturated gain regime.

\subsection{Power fluctuations}

As discussed in the Introduction, one might consider using the monochromatized radiation

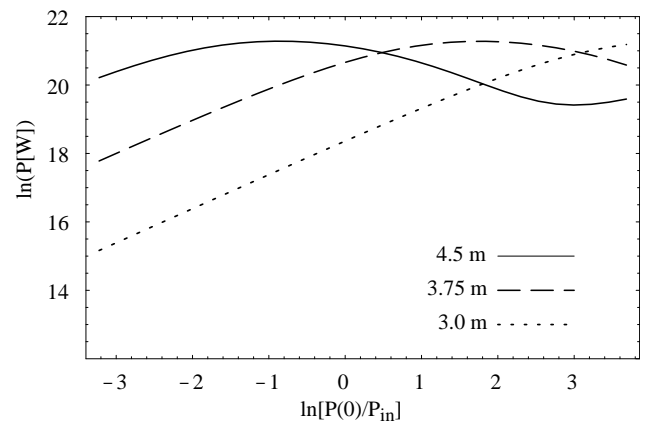

Figure 2. Output power $\ln (P[\mathrm{~W}])$ versus input power (normalized to a nominal input power $P_{\text {in }}$ $=25 \mathrm{~kW}$ ) for several undulator lengths.

produced by a SASE FEL, which will have intrinsic shot-to-shot power fluctuations, to seed a second undulator. In the exponential growth regime (where the first undulator operates), SASE FEL power follows a negative-binomial distribution, characteristic of multimode thermal radiation $[9$, 10]. For a large degeneracy parameter $\langle n\rangle / M \gg$ 1 , where $\langle n\rangle$ is the mean photon number and $M$ the number of modes, the negative-binomial distribution reduces to a gamma distribution

$$
\mathcal{P}(n)=\frac{M^{M}}{\Gamma(M)} \frac{n^{M-1}}{\langle n\rangle^{M}} e^{-M n /\langle n\rangle},
$$

where $\mathcal{P}(n)$ is the probability density and $n$ the photon number. Note that the relative root-mean square (rms) power fluctuations are $1 / \sqrt{M}$ for a gamma probability distribution.

For the split undulator/monochromator scheme, one desires a relatively narrow bandwidth to satisfy user requirements. However, the bandwidth must be large enough to pass sufficient power to greatly exceed the effective shot noise power of the electron beam. Consequently, one will probably operate the monochromator in a regime where $M \lesssim 10$. It then becomes relevant to compute the expected output power fluctuation level of the second undulator given an input power probability distribution from the monochromator which follows Eq. 2. Figure 3 


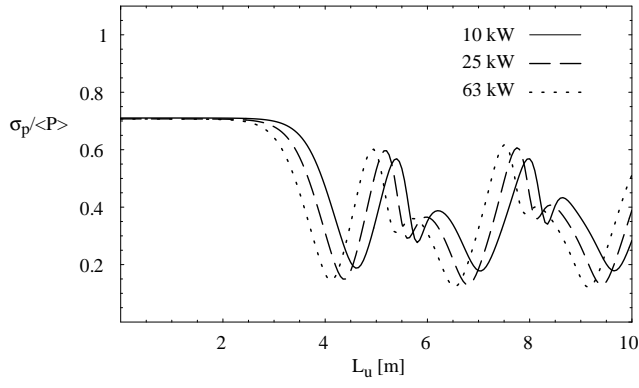

Figure 3. Relative rms output power fluctuation level $\sigma_{P} /\langle P\rangle$ versus undulator length $L_{u}$ for a FEL amplifier whose input power obeys a $(M=2)$ gamma probability distribution.

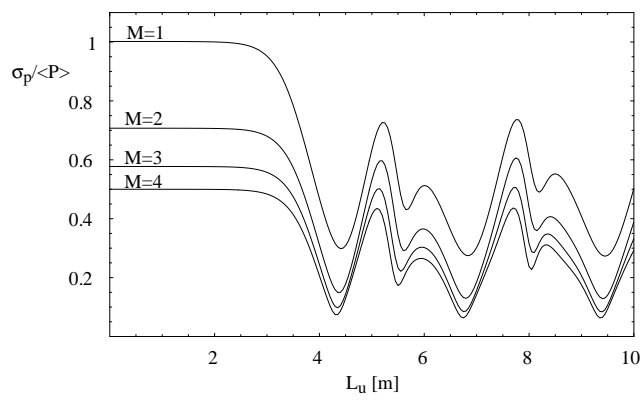

Figure 4. Relative rms output power fluctuation level $\sigma_{P} /\langle P\rangle$ versus undulator length $L_{u}$ for $\left\langle P_{\text {in }}\right\rangle=25 \mathrm{~kW}$ and $M=1,2,3$, and 4 .

shows the normalized rms output power fluctuation $\sigma_{P} /\langle P\rangle \equiv\left(\left\langle P^{2}\right\rangle /\langle P\rangle^{2}-1\right)^{1 / 2}$ versus $L_{u}$ for several mean input powers for the $1 \mathrm{D}$ example of the previous section. These curves were obtained by using a lookup table of $P_{\text {out }}\left(L_{u}, P_{\text {in }}\right)$ produced by $1 \mathrm{D}$ GINGER runs assuming that the initial shot-to-shot power fluctuations obeyed a gamma probability distribution with $M=2$. As the FEL enters the saturated gain regime the relative rms power fluctuations decrease and $\sigma_{P} /\langle P\rangle$ becomes nearly periodic with $L_{u}$, oscillating at the synchrotron bounce period $c \tau_{b}$.

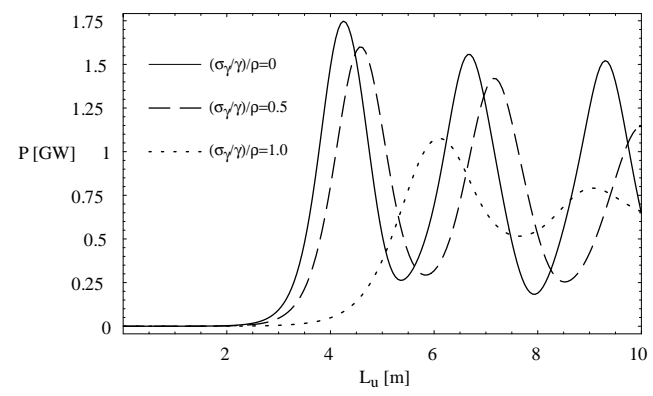

Figure 5. Radiation power $P[\mathrm{GW}]$ versus undulator length $L_{u}$ for several initial energy spreads and an input power $P_{\text {in }}=25 \mathrm{~kW}$.

To examine the sensitivity to the specific probability distribution chosen, we computed similar curves of $\sigma_{P} /\langle P\rangle$ as a function of $L_{u}$ for several different gamma probability distributions $(M=$ $1,2,3$, and 4) for $P_{\text {in }}$ about a mean input power of $25 \mathrm{~kW}$. Figure 4 shows that the general curve shape and the undulator length where the output power fluctuation is minimum appear quite insensitive to the particular choice of input power probability distribution. The first minimum occurs at the first maximum of the mean output power $\left(L_{\text {sat }}=4.25 \mathrm{~m}\right)$, while the second local fluctuation minimum occurs at the minimum of the post-saturation output power, at $L_{u}=5.35 \mathrm{~m}$ ( $c f$. dashed curve of Fig. 1). Consequently, it does appear there is an optimal length for the second undulator to minimize output power fluctuations.

\subsection{Effects of beam energy spread}

The effects of non-zero instantaneous electron beam energy spread $\sigma_{\gamma}$ on the statistical properties of the radiation were also examined. Figure 5 shows the radiation power versus $L_{u}$ for initial uncorrelated energy spreads $\sigma_{\gamma} /(\gamma \rho)$ ranging from zero to one, with input power $P_{\text {in }}=25 \mathrm{~kW}$. Note that the oscillation amplitude and wavenumber decreases as $\sigma_{\gamma}$ increases, presumably due to a much larger spread in synchrotron periods associated with completely filled ponderomotive wells.

Non-zero energy spread also strongly affects the output power fluctuation level for a given input 


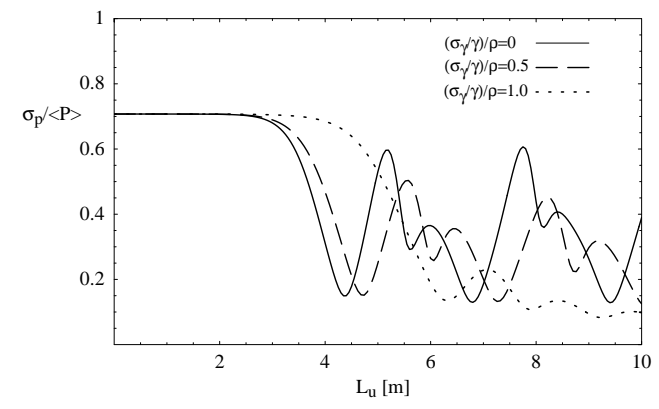

Figure 6. Relative rms power fluctuations $\sigma_{P} /\langle P\rangle$ versus undulator length $L_{u}$ for several initial uncorrelated energy spreads, with $\left\langle P_{\text {in }}\right\rangle=25 \mathrm{~kW}$ and $M=2$.

power probability distribution. Figure 6 plots $\sigma_{P} /\langle P\rangle$ versus $L_{u}$ for different initial uncorrelated energy spreads assuming $\left\langle P_{\text {in }}\right\rangle=25 \mathrm{~kW}$ and $M=2$ ( $c f$. Eq. 2). One sees that an increase in energy spread $\sigma_{\gamma}$ reduces the output power fluctuation level for large undulator lengths, however at a price of increased saturation length and reduced saturation power.

\subsection{D results using LCLS parameters}

A series of FEL amplifier simulations, for standard LCLS parameters [4] $\left(I_{B}=3.4 \mathrm{kA},\langle\gamma\rangle=\right.$ 28077, $\sigma_{\gamma} / \gamma=2 \times 10^{-4}, \lambda_{s}=0.15 \mathrm{~nm}$, and $\varepsilon_{N}=1.2 \pi \mathrm{mm}-\mathrm{mrad}$ ), were performed using GINGER with full transverse physics (i.e., axisymmetric $r-z$ field resolution and full 3D macroparticle motion). Due to the relatively large LCLS emittance, transverse effects are important and act as an additional contribution to energy spread. Using the same methodology as in the above 1D runs, we generated a table $P_{\text {out }}\left(L_{u}, P_{\text {in }}\right)$ and computed $\sigma_{P} /\langle P\rangle$ assuming the input power obeys a gamma probability distribution. Figure 7 shows a family of fluctuation level curves, similar to the $1 \mathrm{D}$ results ( $c f$. Fig. 4), whose minima are nearly independent of the initial fluctuation level $M$.

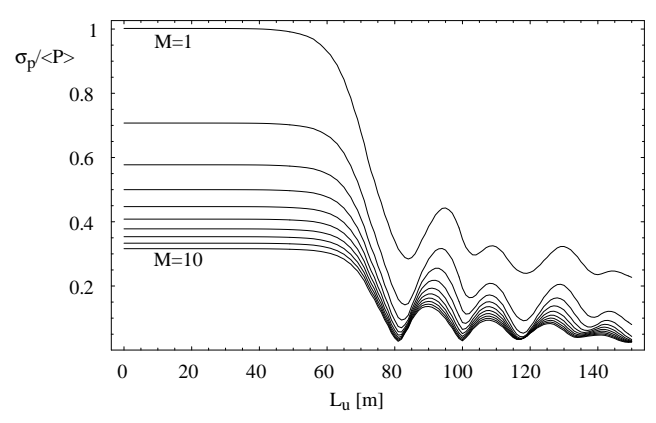

Figure 7. Relative rms output power fluctuations $\sigma_{P} /\langle P\rangle$ versus undulator length $L_{u}$ for $M$ ranging from 1 to 10, using LCLS parameters with initial mean input power of $\left\langle P_{\text {in }}\right\rangle=25 \mathrm{~kW}$.

\section{Conclusions}

This paper has investigated the statistical properties of the expected fluctuation level in output power from an FEL amplifier operating at and beyond saturation where the initial seed radiation comes from a SASE FEL operating in the linear regime. There are well-defined minima in the relative rms output power fluctuation level $\sigma_{P} /\langle P\rangle$ for a given input power and energy spread $\sigma_{\gamma} /(\gamma \rho)$. The location of the minima are insensitive to the initial fluctuation distribution and correlate strongly with power maxima of post-saturation oscillations. Hence, to minimize fluctuations, the undulator length should be chosen to be the location of the first power maximum after saturation. For large electron beam energy spreads [i.e., $\left.\sigma_{\gamma} /(\gamma \rho) \gtrsim 1\right]$, the natural post-saturation fluctuation level is reduced, but, due to the reduced output power and required increase in undulator length, it is unlikely experiments would purposely operate in this regime.

\section{Acknowledgements}

This work was supported by the U.S. Department of Energy under Contract No. DE-AC0376SF0098. Computational resources were provided in part by the National Energy Research Supercomputer Center (NERSC). 


\section{REFERENCES}

1. V. Ayvazyan et al., Phys. Rev. Lett. 88 (2002) 104802.

2. A. Tremaine et al., Phys. Rev. Lett. 88 (2002) 20480.

3. A.H. Lumpkin et al., Phys. Rev. Lett. 88 (2002) 234801.

4. Linac Coherent Light Source (LCLS) Conceptual Design Report, SLAC Report No. SLACR-593, UC-414, 2002.

5. TESLA Technical Design Report, DESY Report No. DESY-2001-011, 2001.

6. J. Feldhaus et al., Opt. Commun. 140 (1997) 341.

7. C.B. Schroeder et al., Pellegrini, S. Reiche, J. Arthur, P. Emma J. Opt. Soc. Am. B 19 (2002) 1782.

8. W.M. Fawley, LBNL Report No. LBNL49625, 2002.

9. E.L. Saldin, E. A. Schneidmiller, M.V. Yurkov, Opt. Commun. 148 (1998) 383.

10. J. Goodman, Statistical Optics (Wiley, New York, 1985). 